\title{
Development of a Low Cost Re-usable Microfibre Sanitary Pad
}

\author{
Christopher Chakwana ${ }^{1}$, Londiwe Nkiwane ${ }^{2}$ \\ National University of Science and Technology, Department of Textile Technology \\ P.O. Box AC 939, Ascot, Bulawayo, Zimbabwe \\ londiwe.nkiwane@nust.ac.zw
}

Received 8 December, 2013; Accepted 24 December, 2013; Published 4 July, 2014

(C) 2014 Science and Engineering Publishing Company

\begin{abstract}
Menstrual hygiene management is a big problem for women and girls in Zimbabwe as the sanitary pads are expensive. An average pack of 8-10 at \$US1.50 is significantly expensive for females coming from low income families, which has caused women and girls to resort to unhealthy ways such as using grass and cow dung for managing their menstruation, which exposes them to infections. The aim was to develop a low-cost re-usable microfibre sanitary pad with good performance properties and low cost maintenance, affordable to poor women. The design was based on the Johnson and Johnson re-usable (a rectangular shape) sanitary pads. The new re-usable pad was developed from polyester microfibre materials. It has a pocket for an insert of another material that will enhance the collection of blood. The developed pad was tested for wicking, leakage and strike through properties. The newly developed pad exhibited better properties and yet cheaper than the commercial disposable pads.
\end{abstract}

\section{Keywords}

Sanitary pads; Pad design; Microfibre; Wicking; Absorption; Strike through; Re-usable

\section{Introduction}

At puberty, girls begin to undergo menstruation; a monthly shedding process of the uterus lining in females of reproductive age. Menstrual periods occur, on average, every 28 days ${ }^{[1]}$. During this time, a woman continuously discharges blood and other chemicals and therefore needs a form of absorbent material, to collect and retain the menstrual flow [2]. Sanitary pads can be used as absorbent materials for the purpose. The pads are both disposable and reusable, and sometimes referred to as, diapers, napkins or towels ${ }^{[3]}$. Menstrual hygiene management is a big problem for women and girls in Zimbabwe as the sanitary pads are expensive. An average pack of 8-10 pads in Zimbabwe costs at least \$US1.50, which is significantly expensive for females coming from low income families. This causes them to resort to crude but inexpensive ways of menstrual management.

Literature shows that bleeding during monthly periods usually lasts for 5-7 days, but can be more or less ${ }^{[4]}$. For 5 to 7 days, one will need about 3 packets of pads which means that she will need at least US\$6 every month, which is a lot of money for the majority unemployed women. A re-usable pad will be more ideal for such a situation, and more so a pad produced from microfibres will be most suitable due to their desirable qualities required for sanitary wear, such as wash-ability and absorbency. Besides, disposable sanitary pads pose environmental concerns as some of the materials used in the pads are non-biodegradable, and thus cause environmental pollution. Disposal of used sanitary products is done either by flushing them out along with the domestic waste where they are later incinerated or deposited and buried in landfills. Incineration is a major cause of pollution worldwide. However, the alternative of burying rubbish in the ground is not much of an improvement because the polyethene plastics used in sanitary pads do not biodegrade, and will remain in the environment unchanged for hundreds of years [5].

Re-usable sanitary pads are not readily available in the Zimbabwean market, while the disposable pads are. Only re-usable pads that have been developed by individuals for personal use or by None Governmental Organisations (NGOs), to assist women especially from the rural areas are available. These sanitary pads made from conventional fibres materials such as cotton and paper ${ }^{[3]}$ are plentiful because these 
materials are readily available. For absorption of blood, materials should combine all the necessary properties required which include; fluid acquisition and its distribution, fluid retention, and washability ${ }^{[3]}$.

\section{Types of Sanitary Pads Available and their Performance}

There are a variety of sanitary pads available locally, some manufactured in Zimbabwe and others imported. The differences among these sanitary pads lie in their methods of manufacture, and levels of performance. Sanitary pads come in a variety of types and thicknesses, comprising of different materials, absorbency properties, shapes and sizes. Their thicknesses vary from the thinnest 'panty liner size' with a thickness of less than $2 \mathrm{~mm}$, to the thickest 'super' size whose thickness is about $10 \mathrm{~mm}^{6}$.

\section{Absorbency}

The panty liners are used during spotting or during high discharge and are not suitable for menstrual management. Absorbency is the characteristic of the pad to take up the blood, and the length of time, it can retain the blood before leakage occurs. Thickness does not always indicate good absorbency[6], but the nature of fibres the pad is made from determines the uptake of blood, as some fibres can be hydrophilic while others hydrophobic. The ultra-thin ( $2 \mathrm{~mm}$ thick) pads use lightweight materials which absorb many times their weight in liquid and can be used for heavy flows. Super-size are overnight pads that are meant to be worn during sleep, but are also used by new mothers and women with extra-heavy flows ${ }^{[6]}$. Super-size pads have higher absorbency due to the more absorbent polymer in the pad.

\section{Shapes}

Disposable sanitary pads used nowadays are designed with an adhesive backing as opposed to the re-usable pads women used in the past; and are of two basic shapes. The first shape of pad is a simple rectangular strip that adheres to the lining of an underwear. The second shape adds side leak protection or wings to that basic shape ${ }^{[6]}$. The second shape has absorbent flaps which fold around the underwear and adhere underneath. The purpose of these flaps is to offer side protection from overflows at the sides so as to minimise staining of the underwear. Both types offer extra-long sizes for extra protection, especially during activities and sports[6].

\section{Materials}

The pads generally found in the market have a plastic material or latex disposable material at the bottom cover. The inside materials range from cotton to absorbent gels. The top layer material differs from manufacturer to manufacturer[6]. The most common types of materials used for the top cover are plain cotton materials and synthetic materials which wick moisture away and leave the wearer feeling drier. The synthetic materials in pads contain latex, which some women are allergic to ${ }^{[6]}$. For that reason, cloth pads are making a comeback. These pads are usually made from cotton or wool-based fabrics and have 'snaptogether' or 'hook-and-loop' wings. Some cloth pad brands have liners, which can be added to the increase in absorption. However, research has shown that microfibres exhibit superior properties required in many textile products including sanitary wear, as compared to conventional fibres, such as cotton and wool[7]. These properties include high liquid absorbency and the ability to hold the liquid within the fibre structure, thus aiding in the prevention of soiling of the outer garment $t^{[7]}$.

Microfibres, can be staple fibres and filaments that have a linear density of between 0.3 and 1.0decitex ${ }^{[8]}$. The microfibres have higher ability to distribute the absorbed fluid within the pad as compared to conventional fibres like cotton. This gives them an added advantage in that they are capable of high fluid retention ${ }^{[8]}$. Microfibres also have a higher wicking ability than cotton which is the most common fibre used in re-usable sanitary pads at the moment (Fig 1).

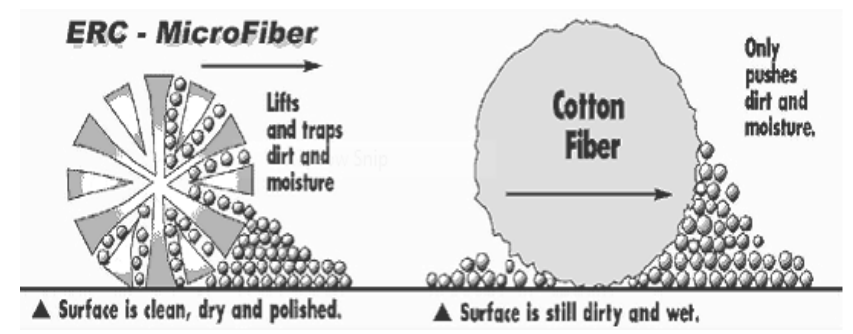

FIG. 1 COMPARISON OF DIRT AND MOISTURE TAKE UP FOR MICROFIBRE AND COTTON [8]

Microfibre fabrics are generally lightweight due to their fineness which helps in the production of light weight sanitary pads. One important point that Purane and Panigrahi ${ }^{[8]}$ highlight, is the ease in which microfibres can be cleaned (washed).This is a very important characteristic for re-usable absorbent articles as they need to be constantly washed [8], especially during menstrual hygiene management. 


\section{Sanitary Pads Currently in Use}

Menstrual pads have been in use as early as the 10th century. Before the advent of disposable sanitary pads, women often used a variety of home-made menstrual pads, crafted from old clothes, grass, or any other absorbent materials they deemed suitable for menstrual management. Disposable sanitary pads were first commercially available from around 1888 with the Southall's pad [9]. The pads started with nurses using wood pulp wrapped in bandages to catch their menstrual flow, thus creating a pad that was made from easily obtainable materials and inexpensive enough to throw away after use ${ }^{[9]}$. Today, women use different methods for menstrual management and these include, modern disposable sanitary pads, tampons, cloth menstrual pads and other alternatives. The choice of these products is based on their income, different lifestyles and careers, activities and the situation they may find themselves in ${ }^{[10]}$. Women from very poor backgrounds resort to using rags, leaves, cow dung and tissues to collect menstrual blood ${ }^{[10]}$. These crude ways expose them to health risks. However, materials used by women who have enough money to purchase sanitary wear, have some short comings in terms of absorbency and fluid retention. This has been a cause of concern as the wearer of the sanitary pads expects good performance in terms of comfort, fit, fluid retention and minimal staining. Sanitary pads should not only possess the required properties for sanitary wear, but be affordable even to the poor. A re-usable sanitary pad is not only more environmentally friendly but is cost effective, and has health benefits as well.

\section{Design of a Re-usable Sanitary Pads.}

Sanitary pads are developed following the basic design as outlined by Mazgaj Yaramenka and Malovanal[11], and in http://www.glamcheck.com/fashion [12]. The pad consists of an outer shell and an insert.

The sanitary pad should be used over and over again and for the purpose that the pad should have re-usable insert and outer shell materials that are all washable (Fig 2). Re-usable pads have usually used a cotton material for insert and outer shell. Most re-usable pads with cotton insert materials have problems of causing leakage for higher-flow days, coupled with the difficulty in washing, as blood stain remains on cotton materials even after wash[5].

\section{Outer Shell of the Pad}

The outer shell of the pad is made up of the top cover, bottom cover and the barrier film. The top cover is a liquid permeable material which is in close contact with the body, which serves to transport the body fluids quickly to the absorbent layer, hence maintaining a dry feeling on the body surface. The bottom cover is the outer part of the sanitary pad. The barrier film prevents the absorbed fluid from leaking and is completely impermeable.

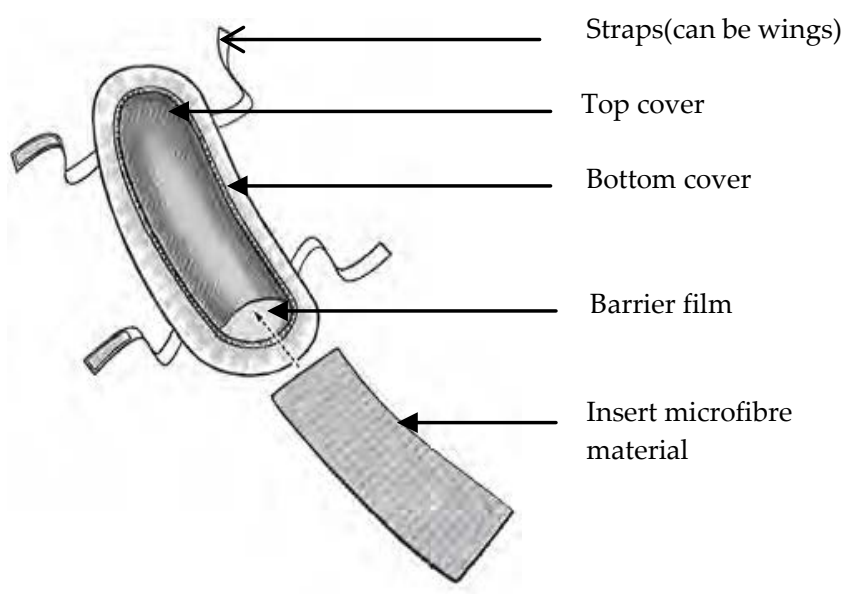

\section{FIG 2 A DIAGRAMMATIC REPRESENTATION OF THE MICROFIBRE SANITARY PAD [11]}

\section{Inner Part of the Pad}

The inner part of the pad is the insert material. Folded cloths are inserted in the pocket of the outer shell.

\section{Fasteners to the pad}

The pad could be fastened to the underwear through the use of wings or straps that fold or tie around one's underwear.

\section{Aims and Objectives}

\section{Aim}

The aim was to develop a low-cost re-usable microfibre sanitary pad with good performance properties and low maintenance.

\section{Objectives}

The objectives of the study were to:

i) Design and develop a re-usable microfibre sanitary pad.

ii) Determine the level of performance of microfibres in the sanitary pads.

iii) Compare the effectiveness of the developed microfibres pad to available brands in the market. 


\section{Methodology}

\section{Development of the Low Cost Re-usable Microfibre Sanitary Pad}

The design measurements on the pad were on the basis of the Johnson and Johnson re-usable pad sample ${ }^{[10]}$. The microfibre re-usable sanitary pad was developed as follows [Fig 3]:

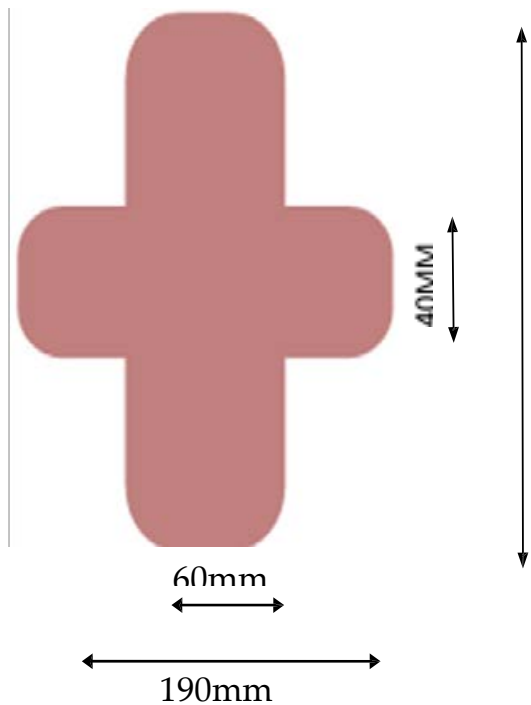

FIG 3 CUTTING THE BOTTOM COVER TO SHAPE USING SPECIFIED MEASUREMENTS

i). The bottom cover of $250 \mathrm{~mm} \times 60 \mathrm{~mm}$, with a wing (65 mm $\times 40 \mathrm{~mm}$ ) on each side, was cut to shape from a woven polyester microfibre material. This material was chosen because it is comfortable to wear close to the skin. The woven structure makes it, strong and yet porous thus enhancing breathability of the sanitary pad $[8]$

ii) A piece of polyethylene film with the same measurements as the bottom cover $(250 \mathrm{~mm} \times 60 \mathrm{~mm})$ was cut from a polyethylene film to create the water proof layer of the pad (cut without wings).The film material was attached to the bottom layer. The polyethylene material is cheap, less bulky, flexible, light and strong enough to withstand forces experienced during normal usage ${ }^{[11]}$.

iii) Another polyester microfibre woven fabric with a pile was then cut using the same measurements of 250 $\mathrm{mm} \times 60 \mathrm{~mm}$, to create the top layer (also cut with wings). The pile loops on the top cover will increase the absorption capacity of the fluid and transport it into the inner absorbent core. The top layer is sewn onto the previous two, with the polyethylene film in between, forming a pocket between the top layer and the polyethylene film. The pocket created is where the insert material that absorbs the fluid will be placed. iv) The insert was made up of a compactly woven polyester microfibre cloth. The compactly woven material was chosen because it will allow gradual flow and uniform distribution of the fluid[13], as compared to other structures such as knitted that are open structures which infringes on fluid retention. An adhesive material was then sewn onto the wing (Figs 4 and 5).

v) To create the adhesive material for the wings, small strips of Velcro material were sewn on each side of the pad wings (Fig 6); used as the adhesive that will fasten the pad to the underwear when worn (Fig 7).

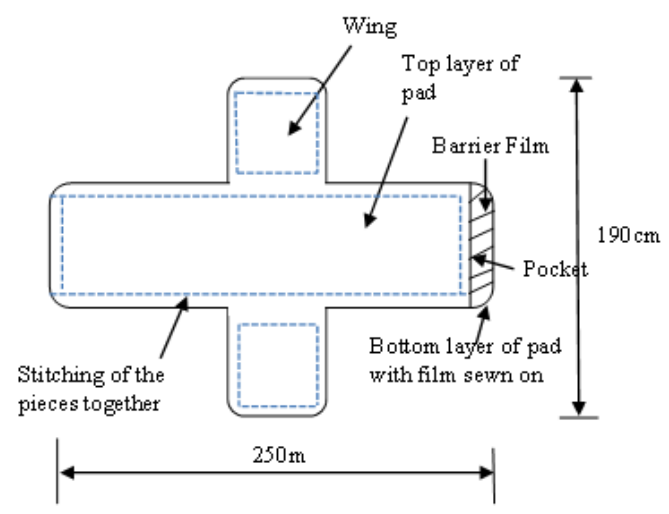

FIG 4 A DIAGRAMMATIC REPRESENTATION OF THE NEW PAD

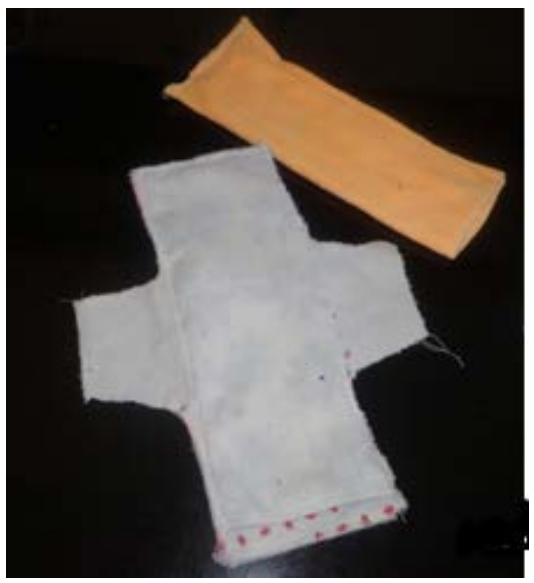

FIG 5 TOP COVER AND THE INSERT MATERIAL

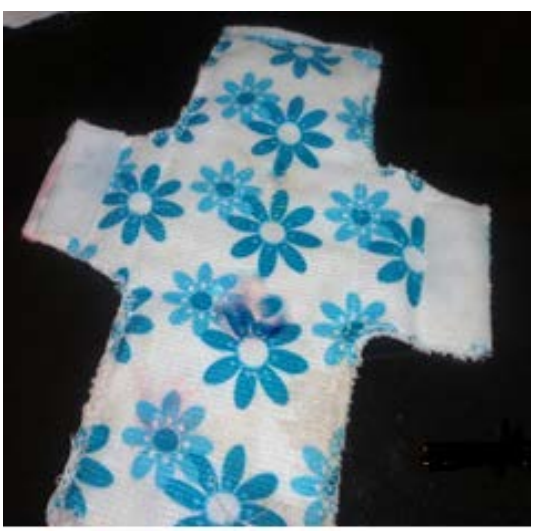

FIG 6 BOTTOM COVER MATERIAL 


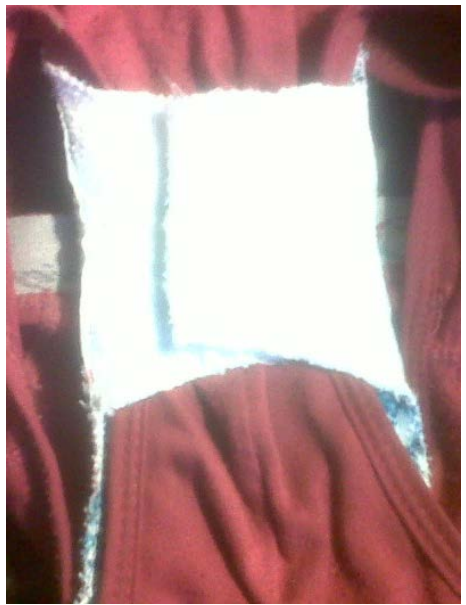

FIG 7 FASTENING OF PAD TO THE UNDER WEAR

\section{Experimentation}

Experiments were conducted on five different thicknesses (ultra-thin which is $2 \mathrm{~mm}$ thick; light 4 $\mathrm{mm}$ thick; regular $6 \mathrm{~mm}$ thick; medium $8 \mathrm{~mm}$ thick; and supper $10 \mathrm{~mm}$ thick) of three different types of sanitary pads, imported commercial sanitary pad, locally manufactured commercial sanitary pad, and the newly designed sanitary pad, in order to compare their functional properties. This resulted in 15 tests being conducted per experiment. Four experiments were conducted using a synthetic blood substitute made from the following ingredients[ ${ }^{[14]}$ :

1. Plain flour $\quad 10 \mathrm{~g}$

2. Distilled water $\quad 200 \mathrm{ml}$

\section{Scarlet food colouring $2 \mathrm{ml}$}

This homogeneous constituency was stored at room temperature during use.

\section{Wicking Experiments}

Wicking experiments were conducted according to BS3424 Method 21(1973) 'Determination resistance to wicking' [15]. This is a vertical strip experiment meant to test the ability of the pad to take up fluid. One end of a sanitary pad was clamped vertically with the dangling end immersed to about $10 \mathrm{~mm}$ in the blood substitute. The fluid absorption along the pad was measured in millimetres after 30 minutes. This method was adopted for all pad samples and the results were then noted ${ }^{[15]}$.

\section{Leakage experiments}

The leakage experiment was conducted using the Cone method test according to EAS 96:2008-Annex B as laid out in the Ugandan Standards Tests for Sanitary
Towels Document[16]. This experiment tested the efficiency of the protective barrier in sanitary pads. Experimental pieces were cut into a square of approximately $6.5 \mathrm{~cm}$ in length from the protective barrier (polyethylene material) and were folded into a cone without creasing, and then placed in a filter funnel which was mounted over an empty beaker. To assess the effectiveness of the polyethylene material as suitable barrier material, the funnel was then filled with the test liquid and left for 48 hours, and the beaker was then inspected to check if any liquid had passed through the water proof barrier material to the beaker[16].

\section{Absorbency Experiments}

Absorbency experiments were conducted according to EAS 96:2008-Annex C, as laid out in the Ugandan Standard Tests for Sanitary Towels Document. These methods were based on measuring changes in weight of the pad using a balance [16], so as to determine the amount of uptake of fluid by the pad. The assessment of the absorption capacity of each pad was made by first finding the dry weight of each class of pad. The initial weight was noted; and the pad then had the test liquid poured on it until saturation was reached (no change in weight following absorption of fluid). On reaching the saturation point, the pad then had a $1 \mathrm{~kg}$ weight placed on it so as to remove the excess fluid within the pad (Fig 8). Excess liquid was blotted from the pad using filter paper and the pad weighed. A load was then applied to simulate the weight of the woman as she sits on the pad during usage. The use of the load also helped to measure the extent to which the protective barrier prevented leakage in the pad ${ }^{[16]}$.

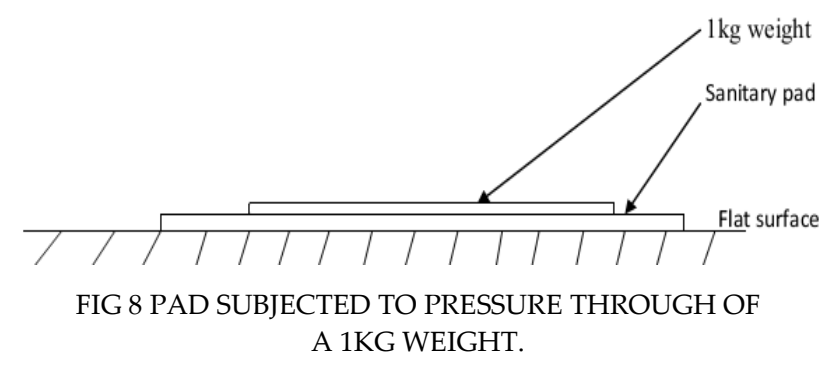

Fluid absorbency was calculated using the following formulas;

Change in mass in grams $(Z)=(X-W) g$

Where $W$ is the initial dry weight of the pad expressed in grams.

$X$ is the final weight of the pad expressed in grams, after absorption of the test liquid. 
$Z$ is the change in mass of the pad after absorption of test liquid.

\section{Strike through Experiment}

The experiment to measure penetration of liquid through the sanitary pad samples was carried out using small volume $(2.0 \mathrm{ml})$ of blood substitute test liquid [17]. A drop of the test liquid was allowed to fall on the pad sample, and the time taken for the liquid to strike through the pad was evaluated by measuring the time taken for the blood substitute to be absorbed from the upper layer of the pad to the inner layer. The drop was closely monitored until the bright red drop of the test liquid appeared like a dull spot on the pad sample, the pads being observed over the same period of time ${ }^{[17]}$.

\section{Results and Discussion}

\section{Wicking Rate of the Sanitary Pad}

The vertical wicking test showed that all the three pads in all five thicknesses, exhibited comparable wicking, with the Microfibre pad's having higher wicking rate than the commercially made sanitary pads.

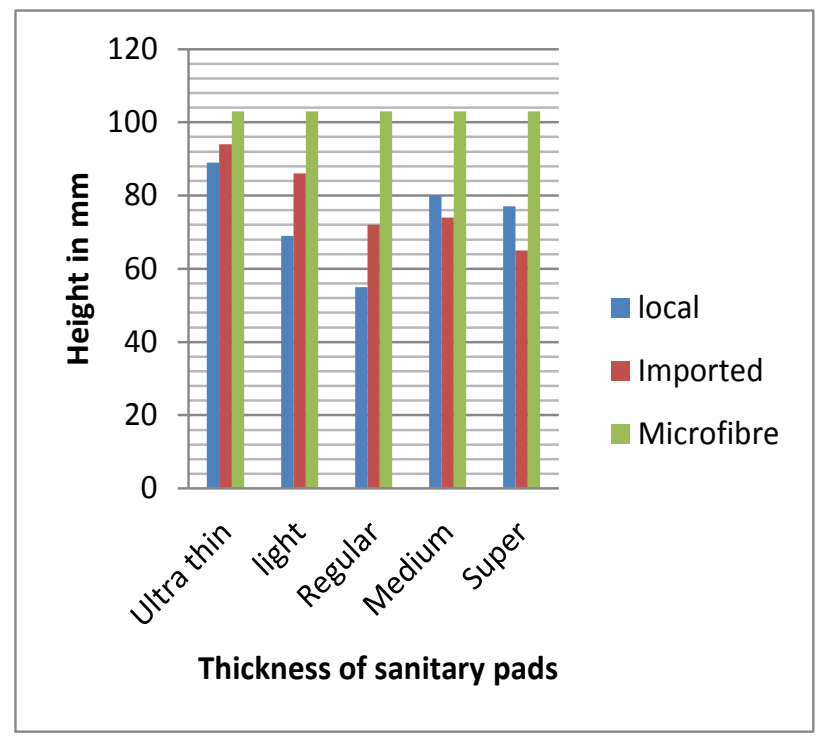

FIG 9 RESULTS FOR THE VERTICAL WICKING EXPERIMENT

It was also noted that with an increase in the pad thickness from the ultra-thin to the super-sized pads, there was a general increase in the rate of wicking. This could be due to the fact that with an increase in pad thickness there was an increase in the amount of super absorbent gels in the absorbent core of the pad. These super absorbent gels were responsible for the transportation of the fluid within the pad. The high wicking values obtained in polyester microfibres are due to their super-absorbency, absorbing over 7 times their weight ${ }^{[8]}$. The microfibres also dry quickly, in one-third of the time of natural fibres, and are environmentally friendly ${ }^{[8]}$. Wicking is a desired characteristic of sanitary pads as they allow the blood to be distributed along the entire structure of the pad and at the same time allowing retention and distribution of the collected blood in the pad, leading to reduction in leaking.

\section{Leakage}

To avoid leakage of blood, a polyethylene material was tested to determine its ability to prevent blood from passing through. Results showed that the polyethylene protective barrier was a useful liquid proof barrier. This was because polyethylene was an impervious continuous film, hence no liquid was collected in the beaker after 48 hours.

\section{Absorption Capacity of the Microfibre Pad as Compared to Commercially Available Pads.}

The absorption capacity the microfibre sanitary pad was compared to that of the two available pads, one locally manufactured and the other imported. These were classified according to their thicknesses from ultra-thin to the thickest super-size.

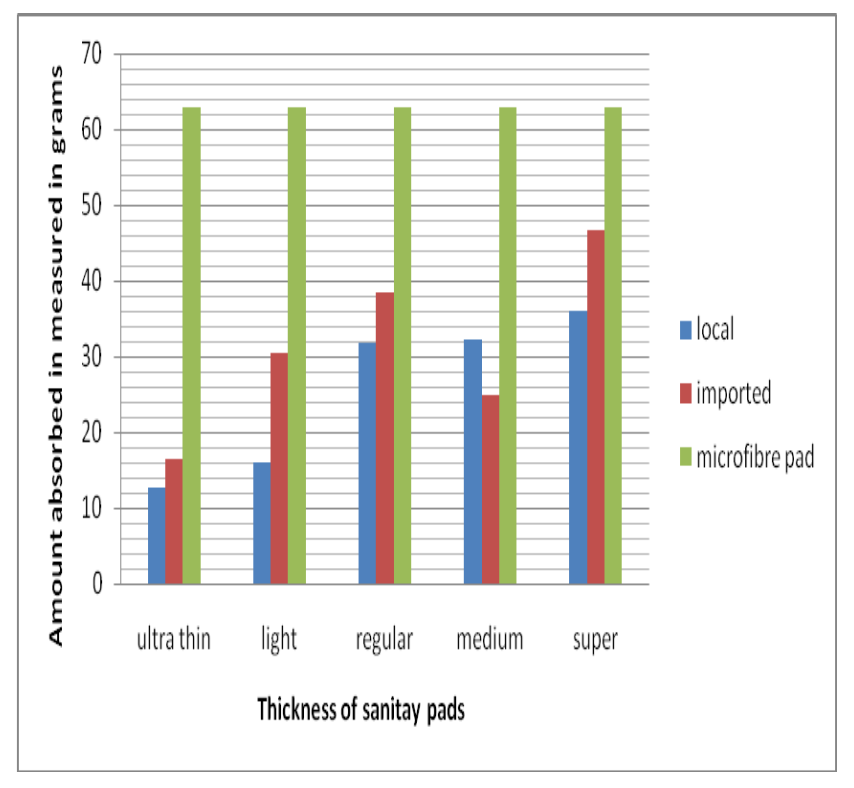

FIG10 ABSORPTION OF TEST FLUID BY THREE DIFFERENT SANITARY PADS

Fig10 shows the sanitary pads absorbent capacity (amount of liquid taken up) when subjected to $1 \mathrm{~kg}$ pressure. The results indicate that the pad made from microfibres has a higher absorption capacity than the commercially made pads and its absorbency is high for all thickness. This is because the microfibres have very 
high absorbency values as compared to natural fibres constituted in commercial pads. The commercial pads' absorption is a result of super-absorbent gels which form the core that is able to absorb and retain liquid under slight mechanical pressure. These gels swell up holding the absorbed liquid in a solid, rubbery state and preventing any leakage, thus helping in making the pad remain dry during usage. Unlike in microfibre pads, the absorbency of the commercial pads, both imported and locally made, is directly related to the thickness of the pads. The thinner the pad, the less absorbent it was. The imported pads performed better than the local brand. This may be caused by an increase in the gelatinous granules within its structure as compared to locally manufactured sanitary pads. In general the best wet comfort is provided by sanitary pads with higher absorption capacity, and low re-wet. The microfibre pad showing the desirable values such as the high absorbency as compared to its commercial counterparts also has an advantage that it is re-usable. This makes the microfibre pad cheaper as each reusable pad can be used for at least two years, because the polyester microfibers pads will be stronger and more durable than the cotton fibres pads that can be used for up to two years [17]. Microfibres dry quickly therefore three can be used during a menstrual cycle. When the pads are checked for leakage, both the microfibre pads and the commercially made sanitary pads will not show any leakage. This is because they all have a barrier layer that prevents leakage to the bottom pad layer.

\section{Liquid Strike through of the Sanitary Pads}

The strike through results shows the rate of fluid transportation from the top part of the pad to the inner collector layer of the pad.

Fig 11 shows that in general the microfibres have a quicker strike through as compared to the commercially made sanitary pads. The locally made sanitary pads exhibited poor strike through properties compared to imported products as they took a longer time for strike through. In an ideal situation, the strike through results in this case indicated the rate at which the liquid was transported from the surface of the pad to the interior of the pad. What accounted for the strike through results was the wicking characteristic of the fibres making up the pads. The high strike through for microfibres sanitary pad was a result of the high wicking behaviour of the microfibres. The wicking properties of the commercial pads increased with increase in thickness, from the ultra- thin type to super-size. This was due to the increase in the quantity of the super absorbent granules that increased within the pad as its thickness also increased. When a pad has high strike through properties; it means that its upper surface readily takes up the blood and keeps the skin feeling dry avoiding any feel of wetness. This feel of dryness contributes to wearing comfort.

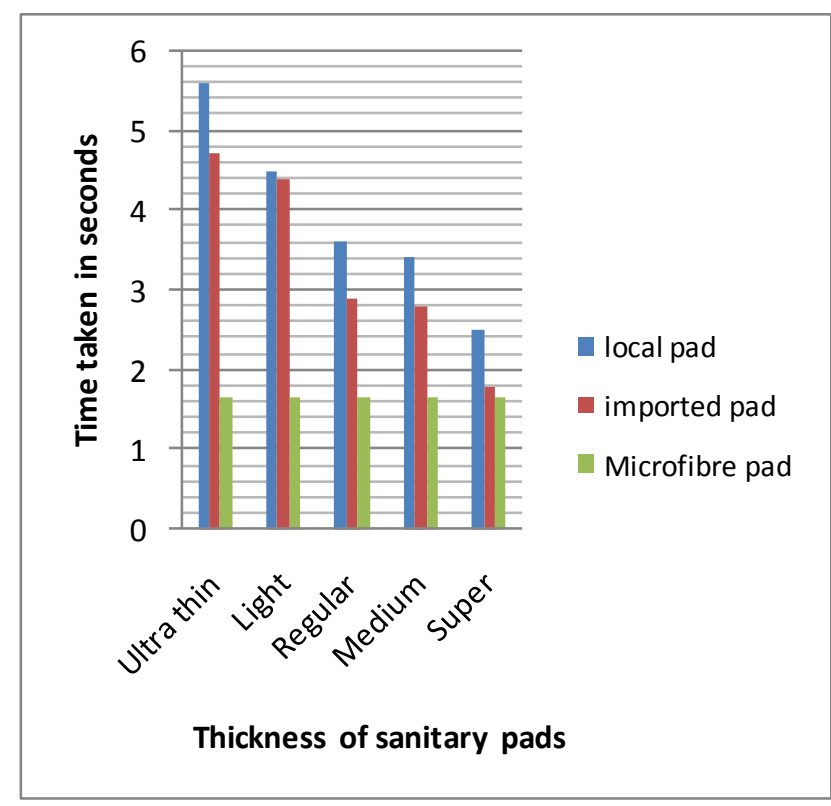

\section{FIG 11 LIQUID STRIKE THROUGH FOR THREE DIFFERENT SANITARY PADS}

\section{Use and Maintenance of the Microfibre Pad}

The newly developed sanitary pad is made from $100 \%$ microfibre. It has a microfibre top cover, bottom cover and insert. The insert is placed in the pocket between the 'covers. The pads are then fastened round the underwear using the Velcro adhesive on the wings of the pad. After use, the insert material is removed from the pad outer shell and both of them are socked in cold and mild salty water. Soaking in cold water minimizes the chances of staining so that the blood does not leave permanent stains. Washing is carried out using detergent or soap to ensure that microbes are eliminated. Both the insert and outer shell are then dried in the sun, and sterilized using ultraviolet (UV) light and stored for future use. Sun rays are natural sterilisers that kill germs and bacteria ${ }^{[17]}$.

\section{Economics of using the Microfibre Pad Compared to the Commercial Brands}

\section{1) Cost of Production Per Sanitary Pad}

Production cost of one microfibre sanitary pad is calculated taking into consideration cost of the 
materials used and labour costs calculated as $20 \%$ of total cost of materials (Table 1)

TABLE 1 PRODUCTION COST OF ONE HOME MADE MICROFIBRE SANITARY PAD

\begin{tabular}{|l|l|}
\hline Details & US \$ \\
\hline $\begin{array}{l}\text { Polyester microfibre material used on each pad(four pieces } \\
\text { measuring } 250 \text { X190 mm at } \$ 2.00 \text { (enough for one bottom } \\
\text { cover, one top cover and at least two inserts) }\end{array}$ & 2 \\
\hline $\begin{array}{l}\text { Cost of washing soap ( } \$ 0.20 \text { worth of soap is required for } 5 \\
\left.\text { litres }{ }^{[19]}\right)\end{array}$ & 0.08 \\
\hline $\begin{array}{l}\text { Polyethylene film- } 60 \mathrm{~mm} \times 250 \mathrm{~mm} \text { (a } 1 \text { metre X } 1.5 \text { metre } \\
\text { material cost } \$ 4.00)\end{array}$ & 0.06 \\
\hline $\begin{array}{l}\text { Total cost of Velcro strips used }-4 \mathrm{~cm}(100 \mathrm{~cm} \text { of Velcro that } \\
\text { cost } \$ 1)\end{array}$ & 0.04 \\
\hline Total cost of materials and maintenance & 1.18 \\
\hline Labour at $20 \%$ of total cost & 0.24 \\
\hline Total cost of pad & $\mathbf{2 . 4}$ \\
\hline
\end{tabular}

\section{2) Comparative Cost Analysis}

Shanmugasundaram and Godwa ${ }^{[17]}$ state that baby diapers and pads from a blend of bamboo and cotton fibres can last up to two years. These cellulosic fibres are prone to microbial attacks, which means that pads from synthetic microfibres will last longer as they are resistant to microbial attacks. The total cost of one re-usable microfibre sanitary will be $\$ 2.40$. The minimum number of three sanitary pads required per month will enable someone to wear one pad while the second one is drying and the third on stand-by. This will cost the woman $\$ 7.20$ for three padsthat will last at least two years. Generally, for commercially available disposable pads, most women can spend $\$ 3.38(£ 2.25$ ) to $\$ 7.5$ (£5) per month on disposable sanitary pads[18], which will amount to between $\$ 40.50$ and $\$ 90.00$ per year. In Zimbabwe the minimum amount spent will be $\$ 36.00$ ( $\$ 1.50 \times 2$ packets/month $X$ 12) per year for locally manufactures and up to $\$ 86.40$ to (\$3.6 X2packets/month X12) for imported pads. The obvious observation is that the microfbre sanitary pad will be far much cheaper than the cheapest disposable sanitary pads. The microfibre pad could be the most suitable as compared to available pads as it is not just cheaper than the disposable pads, but also washes easily and dries quickly as compared to re-usable pads made from cotton. The polyester microfibre is also comfortable close to the skin ${ }^{[13]}$.

The microfibre pad could be a solution to most rural women's need for menstrual hygiene management, whilst placing emphasis on the cost of the product and the drying time. The pad design adopts the use of a polyester microfibre. The nature of materials used for making re-usable pads has an effect on the drying time and subsequently the cost of the product. In this case the cost covers the drying time, pad maintenance and the durability. The amount of time required for a pad to dry, combined with the frequency of replacing soiled pad with a fresh pad, and dictates the number of pads needed to get through an average menstrual cycle. The number of pads included in a package is directly proportional to the retail cost of the product. This is an important consideration, also given that the average consumer has very limited purchasing power. The faster the pads dry, the fewer pieces are needed to be included in a package and therefore the cheaper the total cost of the package. Consequently the re-usable pad is made from synthetic-based material (that is polyester microfibre) rather than cotton due to the significant variance in drying time ${ }^{[13]}$.

The re-usable microfibre sanitary pad also has anadvantage of being highly absorbent with the capacity to quickly distribute the liquid within the pad coupled with minimum leakage ${ }^{[9]}$. The microfibre pad offers performance properties similar to those found in the commercially available disposable brands, and yet affordable. The pad also offers easy maintenance and reduced straining, which are important in cultures where menstruation is associated with social stigma because women and girls can manage their menstruation without being noticed. The ease of washing also helps to eliminate the blood from the pad pieces. Blood remnants in pads can trigger the development of bacteria that may cause an order in women with poor menstrual hygiene management. Besides the polyester, microfibre pad will be more durable than the cotton pad because of its resistance to microbial attack. The developed pad is easy to wear as it sticks to underwear, thus reducing the risk of the pad falling during use.

\section{Conclusion}

The microfibre pads are easy to make as the rural woman can buy most of the material locally and stitch them together.. The total cost of one re-usable microfibre sanitary will be $\$ 2.40$ as shown the Table 1 , and the three sanitary pads will cost $\$ 7.20$. These will last the woman at least two years given the fact that most of the cotton based re-usable sanitary pads, have a lifespan of about two years, the assumption made is 
that the re-usable microfibre sanitary pad will last longer due to the higher strength of the polyester microfibres as compared to cotton. The economics of the pad also lies in the fact that it can be used over and over again, thus making it a cheaper option for most rural women.

\section{REFERENCES}

Crofts T., “Menstruation hygiene management for schoolgirls in low-income countries", Loughborough, UK, Water, Engineering and Development Centre (WEDC). 2012.

Das A., Kothari V.K., Makhija S. and Avyaya K., "Development of high absorbent light weight sanitary napkins" Department of Textile Technology, Indian Institute of Technology, New Delhi, India, Journal of Applied Polymer Science, Vol. 7, pp 1466, 2007.

"Developing an affordable sanitary $\mathrm{pad}^{\prime}$, http//:www. globalgiving.cpfil/2700/Affordable Sanitary_ Pads.pdf [accessed 19 October 2012].

“Developing re-usable sanitary pads" http//:www. globalgiving.com/fil/2700/Affordable Sanitary Pads [accessed 21 October 2012]

Department of Textile Technology", KSR College of Technology, India, Indian Journal of Fibre and Textile Research, Volume 35, pp201-205 September 2010

Dutkiewicz J., "Some advances in non-woven structures for absorbency, comfort and aesthetics", Autex Research Journal, Vol. 2, No3", September 2002.

http://www.glamcheck.com/fashion/2010/05/19/what-aresanitary-pads-types/ [accessed 27 November 2012].

http://www.livestrong.com/article/119329-types-sanitary" (accessed 05 August 2013)

http//:www.patentstorm.us/applications/20080134769/descri ption.html [accessed 25 March 2013] http://www.personalproducts.co.uk/search.html (viewed 01 December 2013),

Mazgaj M., Yaramenka K., and Malovana O., “Comparative Life Cycle Assessment of Sanitary Pads and Tampons", Royal Institute of Technology Stockholm 2006.

"Microfibre technology and its applications", http://www.indiantextilejournal.com/articles/fadetails.as p?id=4328 [accessed 22 November 2012]

Millington. J., “Development of a synthetic blood substitute for use in Forensic science teaching", (MSc Dissertation) London Metropolitan University. [accessed on 24 November 2012].

Purane V.S., and Panigrahi N.R., "Microfibres, Microfilaments \& Their Applications", Department of Textile Technology, SGGS Institute of Engineering \& Technology, Nanded-431606.Maharashtra, India, Autex Research Journal, Vol. 7, No3, September 2007

“Sanitary Napkin", http://en.wikipedia.org/wiki/sanitarynapkin\#Types-of-menstrual-pads [accessed 19 October 2012

Serbiak P.J., Cesco-Cancian A, Fredrick J.K., and Peerenboom R.J., United States Patent number 5,219,341 "Sanitary napkin with a faster transverse wicking absorbent layer to indicate the approach of maximum fluid capacity", June 151993.

Shanmugasundaram O.L., and Godwa R.V.M., "Development and characterisation of bamboo and organic cotton fibre blended baby diapers" Uganda Standard for sanitary towels specification, US EAS 96, Second Edition Reference number US EAS 96:2008 (CUNBS, 2009-09-04.

“The Menstrual Cycle", http:en.wikipedia.org/wiki/ Menstrual-cycle[accessed on the 22 November 2012. 